\title{
Does time spent using social media impact mental health?: An eight year longitudinal study
}

\author{
Sarah M. Coyne \\ Brigham Young University - Provo, smcoyne@byu.edu \\ Adam A. Rogers \\ Brigham Young University - Provo \\ Jessica D. Zurcher \\ Brigham Young University - Provo \\ Laura Stockdale \\ Brigham Young University - Provo \\ McCall Booth

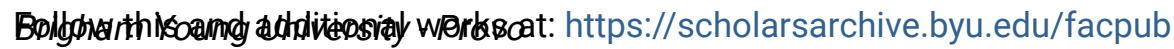 \\ Part of the Other Social and Behavioral Sciences Commons
}

\section{Original Publication Citation}

Sarah M. Coyne, Adam A. Rogers, Jessica D. Zurcher, Laura Stockdale, McCall Booth, Does time spent using social media impact mental health?: An eight year longitudinal study, Computers in Human Behavior, Volume 104, 2020, 106160.

\section{BYU ScholarsArchive Citation}

Coyne, Sarah M.; Rogers, Adam A.; Zurcher, Jessica D.; Stockdale, Laura; and Booth, McCall, "Does time spent using social media impact mental health?: An eight year longitudinal study" (2019). Faculty Publications. 4124.

https://scholarsarchive.byu.edu/facpub/4124

This Peer-Reviewed Article is brought to you for free and open access by BYU ScholarsArchive. It has been accepted for inclusion in Faculty Publications by an authorized administrator of BYU ScholarsArchive. For more information, please contact ellen_amatangelo@byu.edu. 
Full length article

\title{
Does time spent using social media impact mental health?: An eight year longitudinal study
}

\author{
Sarah M. Coyne *, Adam A. Rogers, Jessica D. Zurcher, Laura Stockdale, McCall Booth \\ Brigham Young University, USA
}

\section{A R T I C L E I N F O}

\section{Keywords:}

Social media

Social network

Mental health

Depression

Anxiety

Longitudinal

\begin{abstract}
A B S T R A C T
Many studies have found a link between time spent using social media and mental health issues, such as depression and anxiety. However, the existing research is plagued by cross-sectional research and lacks analytic techniques examining individual change over time. The current research involves an 8-year longitudinal study examining the association between time spent using social media and depression and anxiety at the intraindividual level. Participants included 500 adolescents who completed once-yearly questionnaires between the ages of 13 and 20. Results revealed that increased time spent on social media was not associated with increased mental health issues across development when examined at the individual level. Hopefully these results can move the field of research beyond its past focus on screen time.
\end{abstract}

Does Time Spent Using Social Media Impact Mental Health?: An Eight Year Longitudinal Study

The use of social networking sites (SNSs) are now a normative part of Western adolescent development. Nearly half of adolescents visit SNSs daily, and spend an average of $1 \mathrm{~h}$ per day dedicated solely to social media use (Rideout \& Fox, 2018). However, social media is regularly blamed for the rise in mental health problems in adolescents and young adults (e.g., Charles, 2019), but is social media the real culprit? Or are we engaged in a moral panic, perhaps not understanding the root of the problem? One major limitation of the current literature is that the vast majority of research on SNSs and mental health are cross sectional and cannot speak to developmental change over time or direction of effects. Additionally, research to date rely on traditional regression techniques that model between-person relations among variables. These techniques ignore individual processes that are vital to our understanding of the true relationship between these variables. Thus, the aim of the current study is to test a causal model of the associations between time spent using social media and mental health (anxiety and depression), using both between and within subjects analyses, over an 8-year-period of time, encompassing the transition between adolescence and emerging adulthood.

\subsection{Adolescent social media use, anxiety, and depression}

The development of depression is known as "prototypic multifactorial," or a multi-process syndrome where it is unlikely that "any singular etiological framework (e.g., biological, interpersonal, cognitive, emotional, personality)" provides sufficient explanation for its emergence (Hankin, 2006, p. 105). Research outlines both stressors and vulnerabilities that serve as frameworks to the development of depression (Hankin, 2006). Stressors range from singular, traumatic events to transitional periods (e.g., the onset of puberty) that can perpetuate depressive feelings. Concerning biological sex, girls more often report depressive symptoms than boys in early adolescence, with approximately $25-40 \%$ of adolescent girls exhibiting increased levels of depression compared to $20-35 \%$ of adolescent boys (Petersen, Compas, Brooks-Gunn, \& Stemmler, 1993).

Several studies report associations between increased time spent on SNSs and heightened levels of adolescent depression and anxiety (Banjanin, Banjanin, Dimitrijevic, \& Pantic, 2015; Barry, Sidoti, Briggs, Reiter, \& Lindsey, 2017; Pantic et al., 2012; Woods \& Scott, 2016), particularly among girls (Viner et al., 2019). The link between social media time use and mental health is also evidenced by high profile news articles with headlines such as "Have Smartphones Destroyed a Generation?" (Twenge, 2017b) and "Social Media Linked to Rise in Mental Health Disorders" (Charles, 2019). However, other studies contest connections between adolescent social media time use and associations

\footnotetext{
* Corresponding author. School of Family Life, Brigham Young University, JFSB 2086C, Provo, UT, 84602, USA.

E-mail address: smcoyne@byu.edu (S.M. Coyne).
} 
with anxiety and depression. For example, a number of reviews and a recent meta-analysis suggest that the link between social media and mental health is mixed or tenuous at best (e.g., Best, Maktelow, \& Taylor, 2014; Huang, 2018). Indeed, a recent analysis found that the effect of screen time (including social media) on mental health was as large in effect size as the impact that eating potatoes has on mental health (Orben \& Przybylski, 2019).

\subsection{Theoretical assumptions}

There are a number of theories that might explain associations between time spent on social media and mental health issues during adolescence. For example, the displacement hypothesis (Lin, 1993) suggests that time spent engaging with social media might displace other more important activities that might be protective for mental health, such as sleep (Scott \& Woods, 2018), or face-to-face time with friends (Twenge, 2017a). This theory suggests that time spent with social media might be related to subsequent mental health issues.

Conversely, uses and gratifications theory (Katz, Blumler, \& Gurevitch, 1974) suggests that poor mental health might predict heightened social media use in the future (Bulut \& Dogan, 2017; Quan-Hasse \& Young, 2010). This theory assumes that the individual has a more active role in the media selection process, with each individual choosing to engage in certain types of media to fulfill certain needs. One major motivation for using media in general involves escapism and diversion from everyday life (Coyne, Padilla-Walker, \& Howard, 2013). In this case, an adolescent who is feeling depressed might turn to social media to try relieve depressive symptoms by trying to connect with others online. Alternatively, they may turn to social media as a form of escapism, perhaps to numb emotional pain.

These competing hypotheses suggest several different directions of effects in terms of the relationship between social media and mental health. Direction of effects are rarely examined in this research literature, particularly using longitudinal methods. Accordingly, we will consider and test all possibilities in the current study.

\subsection{Longitudinal studies}

There have been a few longitudinal studies to examine associations between time spent using social media and mental health, with again the results being mixed depending on the study. In some research, social media predicted higher levels of mental health in the future (Coyne, Padilla, Holmgren, \& Stockdale, 2019; Vannucci, Flannery, \& Ohannessian, 2017), while in others, mental health predicted future social media use (Scherr, Toma, \& Schuster, 2018). Additionally, a number of studies have found bi-directional longitudinal relationships between social media and mental health (Frison \& Eggermont, 2017; Houghton et al., 2018; Nesi, Miller, \& Prinstein, 2017). Likely the most influential research on this topic was conducted by Twenge, Joiner, Rogers, and Martin (2018) who examined generational differences regarding media use and a number of adolescent outcomes. The research of Twenge et al. suggests that the current generation (born 1995 or later and termed as "Igen") exhibit greater degrees of depression, loneliness, sleep deprivation, and avoidance to adult roles compared to generations before. These trends appeared around the same time the smartphone became popular, leading Twenge, Joiner, et al. (2018) and Twenge, Martin, et al. (2018) to state that time specifically spent using new media (including social media) might account for the sharp increase in mental health issues among adolescents.

Though these studies have been suggestive of a possible causal link between social media and mental health during development, each have a number of significant limitations. First, the vast majority of longitudinal research is very short in nature, ranging from just a few months to two years in length. Additionally, most research examines only one developmental period, instead of examining social media use across development. However, most important is that almost all the research to date, both cross-sectional and longitudinal, relies on traditional regression techniques that model between-person relations among variables (e.g., rank order correlations and/or change). Developmental questions about causal processes are typically couched at the intra-individual level (including the present question). This means that using between-person methods to draw conclusions about within-person processes is often not a defensible practice, as between- and within-person sources of variability provide fundamentally different types of information (see Berry \& Willoughby, 2016 for a discussion). Thus, within-person analytics are more apt to study such individual level-processes. In other words, if social media really does impact mental health, then we would reasonably expect that adolescents who use more social media would also report higher levels of depression or anxiety. This is the between-person correlation reported in many prior studies. However, we would ultimately need to establish that when adolescents increase their time using social media above their own typical levels, they experience subsequent increases in their own symptoms of depression and anxiety s beyond their own typical levels. Conversely, if an adolescent decreases his/her time spent using social media below his/her typical level, then markers of mental health should decrease for that individual. This prospective, within-person association would more parsimoniously evidence a causal process between social media use and mental health because it is at the level of the individual. Though social media scholars are beginning to examine the within-person level (Boers, Afzali, Newton, \& Conrod, 2019), research has not yet examined longitudinal directionality at this level, which is vital to evaluating the causal model that is often taken for granted in the literature in which social media leads to mental health problems among teens.

Therefore, in the present study, we examined within-person associations between social media time use and mental health using an autoregressive latent trajectory model with structured residuals (ALTSR; Curran, Howard, Bainter, Lane, \& McHinley, 2014). This model effectively disaggregates longitudinal data into its between and withinperson sources of variance and allows for the estimation of withinperson, cross lagged association between these variables. Thus, the current study uses a particularly rigorous technique to examine the longitudinal, bi-directional, within-person associations between social media time use and depression and anxiety, over almost a decade of development and specifically between the transition between adolescence and emerging adulthood. Additionally, given that some studies have found a sex difference in the relationship between social media and mental health (e.g., Viner, et al., 2019), we examine sex as a potential moderator in the current study.

\subsection{The present study}

Disparities across the literatures call for the ongoing study of adolescent social media time use as it relates to the development of anxiety and depression across time and development. We offer three guiding research questions to frame the current research study.

RQ1. Is there a longitudinal, within-person association between the amount of adolescent social media time use and depression?

RQ2. Is there a longitudinal, within-person association between the amount of adolescent social media time use and anxiety?

RQ3. Does adolescent sex moderate the association between time spent using social media and mental health?

\section{Method}

\subsection{Participants}

The participants for this study were taken from the Flourishing Families Project, which is an ongoing, longitudinal study of inner family life involving families with a child between the ages of 10 and 13 
(N 500; $51.6 \%$ female). A power analysis suggested that a sample of at least 500 participants was needed to detect small effect sizes (with a power of 95\%). The data from the current paper is from Waves 3-10, when social media was included in data measurement. There was an $83 \%$ retention rate between all eight years of data collection. At wave 3, participant children averaged 13.82 (SD 1.03) years of age, with mothers averaging 43.1 years in age and fathers 45.3 years. Approximately $67 \%$ of children came from a two-parent family, with $33 \%$ coming from a single parent family. Three hundred and twenty-seven families were of European American ethnicity, 61 were African American, with smaller number for Hispanics (1) and Asian Americans (3). Ninety-eight families are considered multi-ethnic in nature, based on a combination of two or more ethnic cultures among family members. In terms of parental education, $60.2 \%$ of mothers and $47.4 \%$ of fathers had a bachelor's degree or higher. For income categories, $49 \%$ made less than $\$ 25,000$ per year, $28.5 \%$ made between $\$ 25,000$ and $\$ 50,000$ a year, and $22.5 \%$ made more than $\$ 50,000$ per year. Twenty-nine percent of single parents had never been married, $12 \%$ were separated, $52 \%$ were divorced, and $7 \%$ were widowed.

\subsection{Procedure}

Participant families were selected from a large northwestern city and interviewed during the first eight months of 2007 for a Wave I data sample. Wave 3 (the first year in the current paper) took place in 2009. Families were primarily recruited using a purchased national telephone survey database (Polk Directories/InfoUSA). This database claimed to contain 82 million households across the United States and provides detailed information about each household, including presence and age of children. Families identified using the Polk Directory were randomly selected from targeted census tracts that mirrored the socio-economic and racial stratification of reports of local school districts. All families with a child between the ages of 10 and 14 living within target census tracts were deemed eligible to participate in the FFP. Of the 692 eligible families contacted, 423 agreed to participate, resulting in a $61 \%$ response rate. However, the Polk Directory national database was generated using telephone, magazine, and internet subscription reports; as a result, families of lower socio-economic status were underrepresented. Therefore, in an attempt to more closely mirror the demographics of the local area, a limited number of families were recruited into the study via other means (e.g., referrals, fliers; $n \quad 77,15 \%$ ). By broadening the approach, the social-economic diversity of the sample was increased.

All families were contacted directly using a multi-stage recruitment protocol. First, a letter of introduction was sent to potentially eligible families (this step was skipped for the 15 families who responded to fliers). Second, interviewers made home visits and phone calls to confirm eligibility and willingness to participate in the study. Once eligibility and consent were established, interviewers made an appointment to come to the family's home to conduct an assessment interview that included video-taped interactions, as well as questionnaires that were completed in the home. We focus on a number of those questionnaires in the current study. The most frequent reasons cited by families for not wanting to participate in the study were lack of time and concerns about privacy.

We restructured data in the current study by age to address the age range within each wave of data collection. In other words, instead of looking at variables at Wave 3, which had ages ranging from 12 to 16, we restructured the data so variables were considered when all participants were the same age (in this study, age 13, 14, 15, etc.), regardless of the wave at which they were this age. This did result in missing data, particularly at the initial time point with some participants answering the social media questions after age 13 . However, because the age of the child at the first interview was random, data missing at age 13 due to the child not being 13 at the time were missing completely at random, and Mplus' full information maximum likelihood feature was used to deal with these missing values.

\subsection{Measures}

Social media time use. Adolescents responded at all eight waves to one item regarding how many hours they spend in a typical day using social networking sites (e.g., Facebook, Instagram). This was assessed by asking participants, "How much time do you spend on social networking sites, like Facebook, on a typical day?" Response categories ranged from 1 (none) to 9 (more than 8 hours). The item remained the same over the eight years of data collection although the examples changed periodically to reflect more popular social media sites among adolescents.

Depression. Adolescent depression was assessed at all eight waves using the 20-item, self-reported Center for Epidemiological Studies Depression Scale for Children (CES-DC; Weissman, Orvaschel, \& Padian, 1980). Participants responded by rating the degree to which they experienced each item in the past week, with a response scale ranging from 1 (not at all) to 4 (a lot). Sample items included, "I was bothered by things that don't usually bother me," "I felt lonely, like I didn't have any friends," and "I felt like something bad was going to happen." Higher scores indicated greater depressive symptoms. Internal reliability was acceptable ( $\alpha>0.88$ at each wave).

Anxiety symptoms. Anxiety was assessed using the six-item generalized anxiety disorder subscale from the Spence Child Anxiety Inventory (Spence, 1998). Participants responded using a 4-point Likert scale ranging from 0 (never) to 3 (always) with higher scores reflecting greater levels of anxiety. Sample items included, "I worry a lot about things," and "When I have a problem, my heart beats really fast." Higher scores indicated increased levels of anxiety. Internal reliability was acceptable ( $\alpha>0.82$ at each wave).

\subsection{Analytic strategy}

Assessing within-person associations between social networking and mental health. To examine our main hypotheses regarding the within-person associations between adolescents' social networking and mental health indices, we estimated autoregressive latent-trajectory models with structured residuals Curran et al., 2014) following procedures outlined by Berry \& Willoughby (2016). The observed scores on the social networking and mental health variables between ages 13-20 were regressed onto latent growth parameters (e.g., intercept, slope, and quadratic term) and centered at age 13. These parameters represent the random, between-person variability in trajectories of social networking and mental health across adolescence (e.g., initial levels at age 13, rates of change across time). Next, each of these same observed scores are regressed onto their own latent variables, with loadings constrained to one, to form structured residuals. These residuals absorb all remaining within-person variance in the observed scores, and thus represent adolescents' intra-individual fluctuations from their own typical levels of social networking, depression, and anxiety at a specific time point (see Fig. 1 for model specification).

Interpretation of between- and within-person estimates. The presence of both the growth parameters (e.g., intercepts and slopes) and the structured residuals in the same model effectively disaggregates the between and within-person sources of variability from all the observed scores. As such, the associations between social networking and mental health can be estimated at both the between- and within-person levels. At the between-person level, covariances between the random intercepts and slopes indicate the degree to which individual differences in social networking trajectories (initial levels and rates of change) correspond to individual differences in their depression or anxiety trajectories (initial levels and rates of change), similar to a dual process model.

At the within-person level, autoregressive and cross-lagged paths were specified amongst the structured residuals. These are the paths of primary interest to the study because they resemble the causal processes by which social media time use and mental health are ostensibly related 


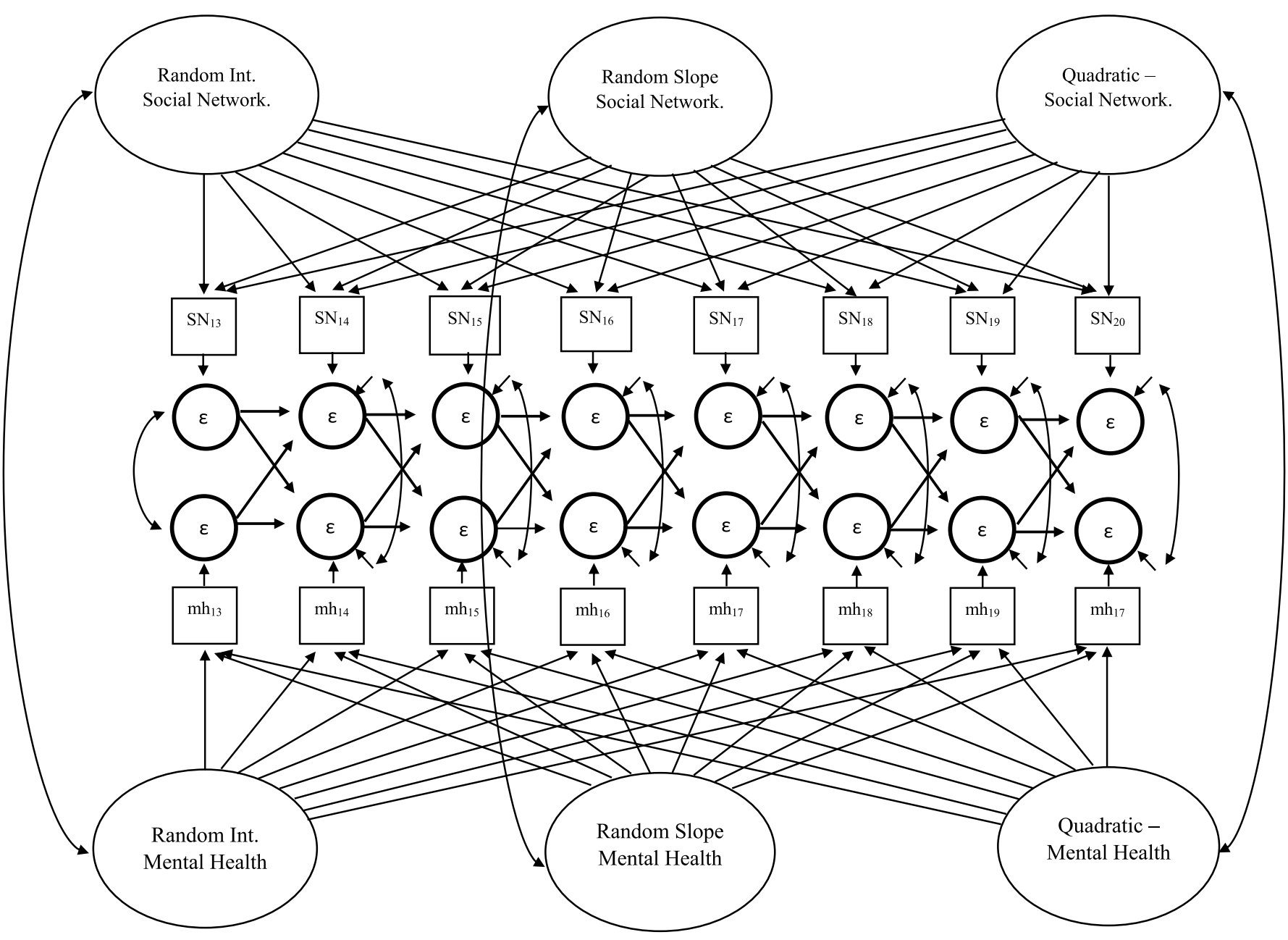

Fig. 1. Specification of ALT-SR model examining between and within person associations between social network and mental health indices.

for the individual adolescent. For example, the lagged path linking social networking to depressive symptoms represents the degree to which an individual's change in social networking beyond his/her typical levels predicts change in his/her typical levels of depressive symptoms at the following time point. Importantly, this association is then adjusted for prior fluctuations in depressive symptoms by the inclusion of autoregressive paths among the structured residuals (deviations in depressive symptoms at time $t$ predicted by deviations in depressive symptoms at time $t-1)$.

Finally, all trait-like and relatively stable individual characteristics (e.g., ethnicity) are controlled by the designed disaggregation of between- and within-person variance. As a result, the current analytic approach is able to provide a relatively rigorous test of a causal model in which social media time use impacts mental health by explicitly modelling individual adolescents' changes in changes in social media time use as a correlate of their ensuing changes in markers of mental health.

Invariance testing. Two models were specified for depression and anxiety, respectively, and invariance testing was used to probe the possibility of a more theoretically parsimonious model to describe the data. In each model, within-person parameters were first tested for invariance across time. This involved estimating a fully unconstrained model where all within-person parameters were allowed to vary freely across each age. Then, in a series of sequential steps, specific elements of the within-person model were constrained to equality across age: autoregressive paths, cross-lagged paths, and within-time residual covariances. Each step produced a slightly more parsimonious model in respect to time, to be compared with the prior model using the change in chi-square statistic. A non-significant change statistic indicates that the more parsimonious model does not significantly worsen model fit over the prior model from which it was derived, thus justifying its retention. In our model building procedures, therefore, constraints that did not worsen model fit were retained; those that worsened model fit were rejected. Once an appropriate model in respect to time was established, it was treated as a baseline model in a multiple group modelling approach to test for invariance across sex. Here, both within- and between-person parameters were tested for invariance, beginning with a fully unconstrained model in regards to sex. Then parameters at the within- (autoregressive, cross-lagged, within-time residual covariances) and then the between-person levels (intercept and slope covariances, intercept and slope latent means) were sequentially constrained to be equal for boys and girls. Constraints that did not worsen model fit were retained; those that worsened model fit were rejected.

\section{Results}

\subsection{Data screening and sources of variance}

Data were first screened for univariate outliers, defined as 3.29 standard deviations from the mean. Across all variables, there were 13 cases that reported outlying values, and as these outlying values often repeated across multiple waves, these cases were removed, yielding a total analytic sample of 487 . To assess the degree of within- and between-person variance in each of our longitudinal measures, we 
calculated intraclass correlations (ICCs). In this context, the ICC represents the proportion of variability in the repeated measures that is attributable to individual differences among adolescents, or the between-person variance. ICCs were calculated for adolescents' selfreported social networking (ICC 0.54), depressive symptoms (ICC 0.54), and anxiety (ICC 0.57), indicating that between $54 \%$ and $57 \%$ of the variance in these repeated measures was due to trait-like differences among adolescents. This also means that a substantial amount of remaining variance in these measures (between $43 \%$ and $46 \%$ ) can be attributed to within-person fluctuations from wave to wave. As such, the use of the ALT-SR model is justified in order to disaggregate these between- and within-person sources of variability.

\subsection{Descriptive statistics}

Means, standard deviations, and correlations among the key study variables were calculated to identify preliminary data patterns (see Tables 1 and 2). At age 13, adolescents reported an average social networking use of about $31-60 \mathrm{~min}$ per day. These average levels increased fairly steadily so that by young adulthood, they were reporting upwards of $2 \mathrm{~h}$ per day. Notably, there were small to moderate sex differences at each wave, with girls reporting significantly more time on social networking sites than boys. Adolescents also reported low average levels of depressive symptoms and anxiety, but these mean levels also increased throughout adolescence. Moderate to large sex differences characterized these variables at each assessment, with girls reporting higher depressive symptoms and anxiety than boys. Finally, within-time correlations revealed a general pattern of positive association between the social networking variables and mental health indices (see Table 2). These correlations appeared to be somewhat more consistent for girls than for boys from wave to wave.

\subsection{Social networking and depressive symptoms}

Adolescents' time spent using social media was examined in relation to their depressive symptoms across ages 13-20 using an ALT-SR model. Model building procedures found that none of the within-person parameters could be considered invariant across time, and thus all were allowed to vary freely across assessments (see Table 3 for results of invariance constraints). Then, this model was specified in a multiple group framework to test for sex differences. Most within-person parameters were invariant across sex, with the exception of the autoregressive paths for depressive symptoms. At the between-person level, intercept covariances and latent means for the growth parameters did vary among boys and girls. In sum, the most parsimonious and wellfitting model was one in which the within-person processes between social networking and depressive symptoms varied across time but not across sex, and where between-person growth trends did vary across sex. This final model showed adequate fit to the data, RMSEA .055 [95\% CI: 0.04, 0.06]; CFI 0.94; TLI 0.93 .

Results at the between-person level indicated that boys and girls reported low initial levels of social networking and depressive symptoms (intercepts) that increased steadily across adolescence (positive linear slopes), and slightly tapered after peaking around age 18 (negative quadratic term). Among girls, there was a positive covariance between the age-13 intercepts, but no covariance among the slope or quadratic terms. Interpreted, girls' initial levels of social networking at age 13 were significantly and positively correlated with their depressive symptoms, but their rates of change were unassociated. Among boys, there was no significant covariance among the intercepts, but there was a positive and significant covariance in the slope. Although boys' initial levels of social networking and depressive symptoms at age 13 were unrelated, the rate at which they changed in their social networking covaried with their change in depressive symptoms. See Table 4.

At the within-person level, associations among the cross-lagged paths were of primary interest (see Fig. 2a). At all assessments, boys' and girls' social networking was unrelated to their future depressive symptoms. That is, adolescents' increases in their social networking beyond their own typical levels could not explain their changes in depressive symptoms a year later. Likewise, depressive symptoms were generally unrelated to future social networking. Adolescents' fluctuations in depressive symptomology beyond their typical levels do not predicted their later fluctuations in social networking. There was only one exception to this, in which age 16 depressive symptoms predicted lower social networking at age 17 (see Fig. 2).

\subsection{Social networking and anxiety}

In the ALT-SR model for anxiety, model building procedures indicated that the cross-lagged paths between time spent using social media and anxiety were invariant across time, but the auto-regressive and within-time residual covariances were not. Then, results of multiple group modelling indicated that all within-person parameters were invariant across sex. At the between person level, covariances were invariant across sex as well, but the latent means were significantly different among boys and girls (see Table 3). Thus, the final model was one in which the cross lagged paths were invariant across time and sex, but where growth trends (intercepts, slopes) were distinct for boys and girls. This model displayed adequate fit to the data, RMSEA 0.05 [95\% CI: 0.04, 0.06], CFI 0.94, TLI 0.94.

Similar to the model on depressive symptoms, the between person results indicated low initial levels of social networking and anxiety, which increased steadily across adolescence (positive linear slope) until peaking around age 18 (negative quadratic term). There was a significant and positive association between the intercepts, indicating that individuals higher in social networking at age 13 also showed higher average levels of anxiety at age 13. There was also a significant and positive correlation between the slopes. Adolescents who increased more rapidly in their social networking across adolescence tended to also increase more rapidly in their anxiety symptoms. See Table 4.

At the within-person level, however, there were no significant crosslagged associations between social networking and anxiety (See Fig. 2b).

Table 1

Means and standard deviations for all study variables.

\begin{tabular}{|c|c|c|c|c|c|c|c|c|c|}
\hline & \multicolumn{9}{|c|}{ Age } \\
\hline & & Age 13 & Age 14 & Age 15 & Age 16 & Age 17 & Age 18 & Age 19 & Age 20 \\
\hline \multirow[t]{2}{*}{ Social Network. } & $M$ & $2.99(2.42)$ & $3.41(2.86)$ & $3.65(3.06)$ & 3.97 (3.17) & $4.02(3.21)$ & $4.14(3.30)$ & $3.94(3.27)$ & $4.13(3.26)$ \\
\hline & $S D$ & $2.06(1.66)$ & 2.04 (1.79) & 1.78 (1.74) & 1.95 (1.72) & $1.96(1.76)$ & 1.99 (1.76) & $1.71(1.68)$ & 1.83 (1.86) \\
\hline \multirow[t]{2}{*}{ Depressive Sym. } & $M$ & $1.62(1.50)$ & $1.73(1.60)$ & $1.83(1.60)$ & $1.91(1.70)$ & $1.94(1.66)$ & $1.87(1.68)$ & $1.86(1.67)$ & 1.94 (1.67) \\
\hline & $S D$ & $0.49(0.43)$ & $0.55(0.49)$ & $0.60(0.51)$ & $0.67(0.57)$ & $0.64(0.50)$ & $0.63(0.54)$ & $0.59(0.50)$ & $0.66(0.51)$ \\
\hline \multirow[t]{2}{*}{ Anxiety } & $M$ & $0.96(0.70)$ & $1.06(0.76)$ & $1.18(0.78)$ & $1.30(0.89)$ & $1.37(0.95)$ & $1.45(0.96)$ & $1.44(1.02)$ & $1.43(0.99)$ \\
\hline & $S D$ & $0.55(0.49)$ & $0.59(0.50)$ & $0.64(0.50)$ & $0.67(0.51)$ & $0.66(0.57)$ & $0.67(0.62)$ & $0.70(0.63)$ & $0.73(0.62)$ \\
\hline
\end{tabular}

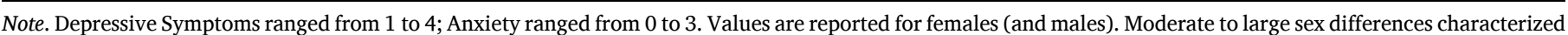

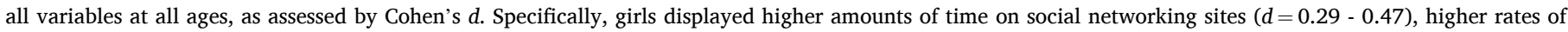
depressive symptoms ( $d \quad 0.25$ - 0.49) and higher levels of anxiety ( $d \quad 0.50-0.76)$ from ages $13-20$. 
Table 2

Within-time correlations among primary study variables from ages 13-20.

\begin{tabular}{|c|c|c|c|}
\hline & $\begin{array}{l}\text { Social Networking } \\
13 / 14 / 15 / 16 / 17 / 18 / 19 / 20\end{array}$ & $\begin{array}{l}\text { Depressive Symptoms } \\
13 / 14 / 15 / 16 / 17 / 18 / 19 / 20\end{array}$ & $\begin{array}{l}\text { Anxiety } \\
13 / 14 / 15 / 16 / 17 / 18 / 19 / 20\end{array}$ \\
\hline $\begin{array}{l}\text { Social } \\
\text { Networking }\end{array}$ & - & $.30^{* * *} / .19^{* *} / .24^{* * *} / .26^{* * *} / .06 / .05 / .26^{* * *} / .26^{* * *}$ & $.06 / .11 / .19 * * / .17 * * / .06 / .08 / .24 * * * / .33^{* * *}$ \\
\hline Depressive Sym. & $\begin{array}{l}-.01 / .12 / .17 * / .10 / .16 * / .05 / .07 / \\
.11\end{array}$ & - & $\begin{array}{l}.38^{* * *} / .50 * * * / .59 * * * / .51 * * * / .52 * * * / .55^{* * *} / .55^{* * *} / \\
.60 * * *\end{array}$ \\
\hline Anxiety & $\begin{array}{l}-.04 / .13 / .21 * * / .09 / .13 * / .09 / .17 * / \\
.19\end{array}$ & $\begin{array}{l}.39 * * * / .45 * * * / .35 * * * / .39 * * * / .41 * * * / .39 * * * / .52 * * * / \\
.46\end{array}$ & - \\
\hline
\end{tabular}

Note. Girls' correlations are represented above the main diagonal; boys' correlations are below the main diagonal.

${ }^{*} p<.05 ; * * p<.01 ; * * * p<.001$.

Table 3

Results of model building procedures in each ALT-SR model testing invariance constraints across time and then across sex.

\begin{tabular}{|c|c|c|c|c|c|c|}
\hline \multirow[t]{2}{*}{ ALT-SR Models } & \multicolumn{3}{|c|}{ Time Invariance } & \multicolumn{3}{|l|}{ Sex Invariance } \\
\hline & $\Delta \chi^{2}$ & $d f$ & $p$ & $\Delta \chi^{2}$ & $d f$ & $p$ \\
\hline \multicolumn{7}{|l|}{ 1. Depressive Symptoms } \\
\hline Autoregressive paths & 24.45 & 12 & $.02^{a}$ & 18.07 & 8 & $.02^{a}$ \\
\hline Cross-lagged paths & 22.34 & 12 & $.03^{a}$ & 10.50 & 8 & .23 \\
\hline Within-time covariances & 41.87 & 7 & $<.001^{a}$ & 19.94 & 8 & $.01^{a}$ \\
\hline Latent covariances & & & & 10.65 & 2 & $.005^{a}$ \\
\hline Latent means & & & & 12.43 & 4 & $.01^{a}$ \\
\hline \multicolumn{7}{|l|}{ 2. Anxiety } \\
\hline Autoregressive paths & 22.81 & 12 & $.03^{a}$ & 14.29 & 8 & .08 \\
\hline Cross-lagged paths & 14.70 & 12 & .26 & 0.20 & 2 & .90 \\
\hline Within-time covariances & 17.12 & 7 & $.02^{a}$ & 8.07 & 8 & .43 \\
\hline Latent covariances & & & & 2.41 & 2 & .29 \\
\hline Latent means & & & & 44.32 & 4 & $<.001$ \\
\hline
\end{tabular}

Note. Superscripts denote constraint that worsened model fit at $\mathrm{p}<.05$, which were relaxed in final models.

Table 4

Between-person parameter estimates and fit indices for final models.

\begin{tabular}{|c|c|c|c|c|}
\hline \multirow{3}{*}{$\begin{array}{l}\text { Parameter } \\
\text { Estimate }\end{array}$} & \multicolumn{4}{|c|}{ Adolescents' Mental Health Indices } \\
\hline & \multicolumn{2}{|c|}{ Depressive Symptoms } & \multicolumn{2}{|l|}{ Anxiety } \\
\hline & Estimate & SE & Estimate & SE \\
\hline \multicolumn{5}{|l|}{ Latent Means } \\
\hline $\mathrm{SN}_{\text {intercept }}$ & $2.93^{* * *}\left(2.72^{* * *}\right)$ & 0.14 & $2.94 * * *(2.72 * * *)$ & .14 \\
\hline $\mathrm{MH}_{\text {intercept }}$ & $1.63^{* * *}\left(1.57^{* * *}\right)$ & 0.04 & $0.91 * * *(0.79 * * *)$ & .04 \\
\hline $\mathrm{SN}_{\text {slope }}$ & $0.42^{* * *}\left(0.39^{* * *}\right)$ & 0.07 & $0.42 * * *(0.37 * * *)$ & .07 \\
\hline $\mathrm{MH}_{\text {slope }}$ & $0.10^{* * *}\left(0.08^{* * *}\right)$ & 0.02 & $0.16^{* * *}\left(0.12^{* * *}\right)$ & .02 \\
\hline $\mathrm{SN}_{\text {quad }}$ & $\begin{array}{c}0.04^{* * *} \\
\left(0.04^{* * *}\right)\end{array}$ & 0.01 & $\begin{array}{c}0.04 * * * \\
(0.04 * * *)\end{array}$ & .01 \\
\hline $\mathrm{MH}_{\text {quad }}$ & $\begin{array}{c}0.01^{* * *} \\
\left(0.01^{* * *}\right)\end{array}$ & 0.01 & $\begin{array}{c}0.01^{* * *} \\
\left(0.01^{* * *}\right)\end{array}$ & .01 \\
\hline \multicolumn{5}{|l|}{ Covariances } \\
\hline $\mathrm{SR}_{\text {int }} \mathrm{W} / \mathrm{PC}_{\text {int }}$ & $.18^{* * *}(.02)$ & $.04(.03)$ & $.07 *$ & .07 \\
\hline $\begin{array}{l}\mathrm{SR}_{\text {slope }} \mathrm{W} / \\
\mathrm{PC}_{\text {slope }}\end{array}$ & $-.001\left(.003^{*}\right)$ & $\begin{array}{l}.002 \\
(.002)\end{array}$ & $.003^{*}$ & .22 \\
\hline \multicolumn{5}{|l|}{ Model Fit } \\
\hline RMSEA & .06 & & .05 & \\
\hline CFI & .94 & & .94 & \\
\hline TLI & .93 & & .94 & \\
\hline
\end{tabular}

Note. SN Social Networking; $\mathrm{MH}$ - mental health indicator; int latent intercept (centered at age 13); slope latent linear growth term; quad latent quadratic growth term. Parentheses given when parameter is non-invariant by sex, with boys' values in parentheses.

$* * * p<.001,{ }^{* *} p<.01, * p<.05, p>.10$.

Social networking did not predict future anxiety. That is, adolescents' increases in social networking beyond their typical levels did not predict intra-individual changes in anxiety one year later. Similarly, anxiety did not predict future social networking: fluctuations in anxiety beyond an individual's typical levels held no predictive power over their future changes in social networking.

\section{Discussion}

The current study examined associations between early adolescence to emerging adult SNSs time usage to depression and anxiety across an eight-year timespan. In general, between subjects analyses (both at the cross-sectional and longitudinal level) revealed that time spent using SNSs was moderately related to anxiety and depression (though there were some differences depending on the sex of participant). These findings corroborate a host of studies suggesting that time spent using social media is related to mental health issues (e.g., Barry, et al., 2017; Lin, Sidani, Shensa, Radovic, Miller, Colditz, 2016). These findings would appear to give support to the displacement hypothesis.

However, the within-person, cross lagged estimates painted a very different picture. Specifically, there were no associations between time spent using social media and mental health across eight years, spanning early adolescence into young adulthood. That is, when individual adolescents used more social media than their own cross-time averages, they did not increase in either depression or anxiety. Similarly, decreases in an individual adolescents' own time spent on social media did not indicate ensuing decreases in depression or anxiety. These findings are at odds with much of the research literature, but do seem to echo assertions from some cross-sectional studies suggesting that the reported link between social media and mental health might be somewhat exaggerated (e.g., Orben \& Przybylski, 2019).

The presence of correlations at the between person level, but their absence at the within-person level, is a critical contribution of the present study. Especially of late, methodologists have voiced concern over using between-person analyses to make assumptions about withinperson change processes (Hamaker, Kuiper, \& Grasman, 2015). This work demonstrates quite clearly that between- and within-person sources of information speak to distinct processes and therefore, answer different research questions altogether (see Fig. 3). Our betweenperson results converge with prior research to indicate who among our sample is higher in mental health difficulties: those who are using social media more often. The within-person results, however, challenge a 
(a)

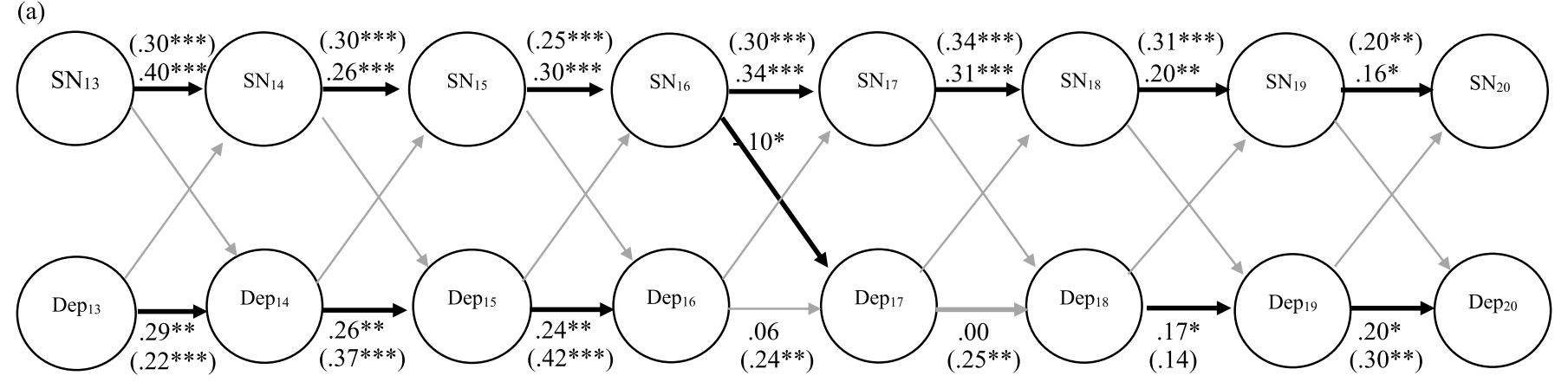

(b)

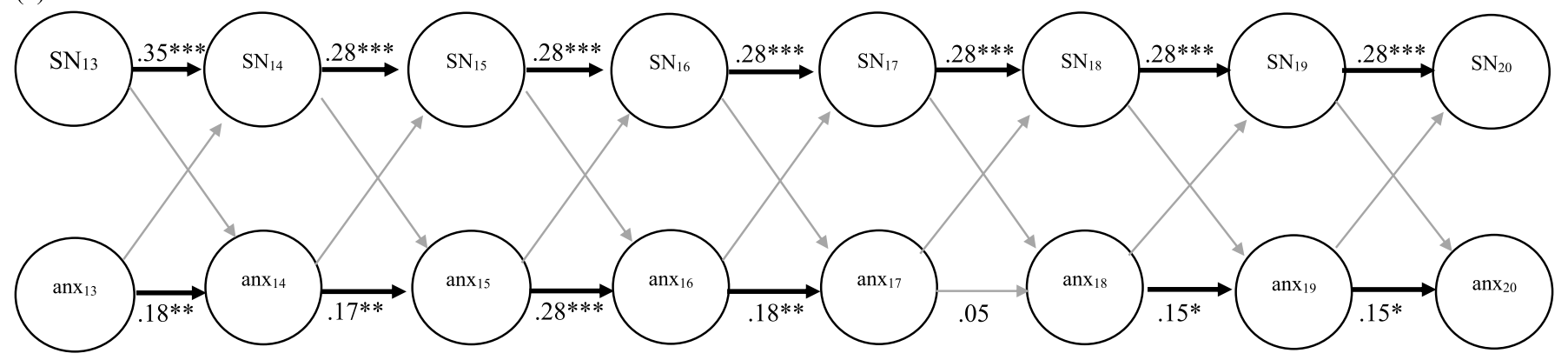

Note. Bolded paths represent significant associations. For simplicity, within-time residual covariances are not shown, nor are any between-person parameters (e.g., intercepts, slopes). When differing by sex, path estimates are displayed for girls (and boys).

$* * * p<.001, * * p<.01, * p<.05$

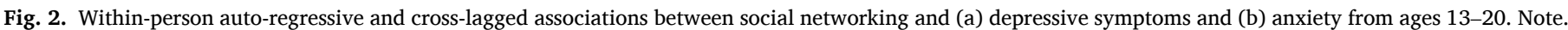

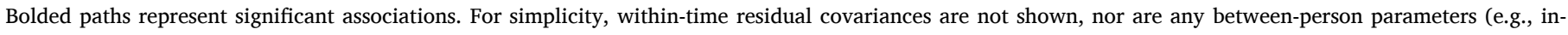
tercepts, slopes). When differing by sex, path estimates are displayed for girls (and boys). ${ }^{* * *} \mathrm{p}<.001,{ }^{* *} \mathrm{p}<.01$, *p $<.05$.

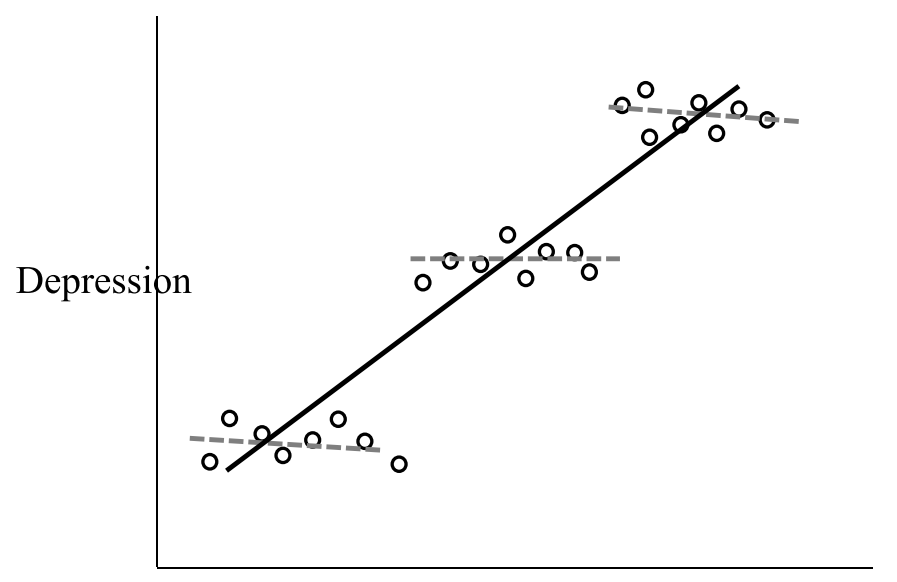

\section{Social Media Use}

Fig. 3. Demonstration of how between- and within-person sources of information can be disconnected. A between person-correlation (bold line) fails to capture within-person correlations and/or heterogeneity of three adolescents assessed at eight time points (dashed lines through eight points).

causal connection between the two, as adolescents' changes in one (e.g., social media use) do not reliably predict changes in the other (e.g., depression). In other words, although adolescents higher in social media time use have more mental health challenges (in general), their own, individualized fluctuations in social media use do not covary with fluctuations in mental health. This suggests that there may be other processes at play, involving extraneous or confounding variables, that more than likely account for these between-person associations. This fits well with a clinical viewpoint of depression where mental health is viewed as a multi-process syndrome where no one stressor is likely the only cause of a significant depressive episode (e.g., Hankin, 2006). Similarly, the development of depression and anxiety are multifactorial, involving person centered characteristics (such as biological predispositions, differences in cognitive control and processing, coping mechanisms), and situational factors (such as chronic stress in early childhood, experiencing traumatic events, continued and prolonged exposure to stress, and sleep). While researchers have argued that time spent social media use may be a social risk factor to the development of depression and anxiety, this does not appear to be the case, at least in the present sample.

Notably, our sample was born between 1993 and 1997, with the median year being 1995 - exactly the year "iGen" began, making this particular sample both relevant and timely. Our findings lead us to wonder if society is in a moral panic, at least concerning the sheer amount of time spent on social media and mental health. We find no evidence that time spent on social media is "destroying a generation", a question that has been tackled in recent years, specifically in reference to smartphones (Twenge, 2017b). Time is an important factor when examining social media and outcomes (especially when applied to theories like the displacement hypothesis), and much of the research literature on this topic has focused on time use. However, we recognize that time use is not the whole story. We hope this study helps the research community and society as a whole to move beyond the screen time debate and instead to examine the context and content surrounding social media use.

While the current study addresses important gaps in the current 
literature, and has a number of strengths, including a rigorous analytic technique, eight years of data across a major developmental transition, and a high retention rate, it is not without limitations. The current study used a single, self-report, item of time spent using social media. In general, research suggests that self-report time on smartphones is only moderately corrected with actual use, with most individuals underreporting their use (Andrews, Ellis, Shaw, \& Piwek, 2015; Lin, Lin, Lee, Lin, Lin, Chang, 2015). Thus, we suspect that reported social media use may be somewhat under-reported in the current sample (though notably it would be under-reported for everyone and general patterns would remain the same). More recent research is beginning to examine social media time use using passive sensing (e.g., apps installed on mobile phones that track real-time usage of mobile phones and phone apps; Meyer et al., 2019). This methodology did not exist when we began the study many years ago. We encourage researchers to utilize this method to examine the long-term impact of social media use on adolescents. Additionally, this was a non-clinical sample and participants self-reported their depression and anxiety. Though this is a common method to assess mental health, there may be some bias when using this method of reporting. Future research could obtain a more clinically based and professional way of assessing mental health. Finally, though the current study is the longest existing study to date on social media and mental health (to our knowledge), social media may have a lingering impact on mental health that might appear later on in life, during a time of life our study did not examine.

In summary, our results revealed that there is no evidence that time spent using social media might influence an individual's mental health over time. We hope these results allow the discussion regarding social media and mental health to move beyond focusing on time, as it appears that time on social media itself is not necessarily indicative of mental health issues. Instead, we hope that researchers examine the context and content surrounding social media use and many other factors that might explain the increase in mental health problems during adolescence and emerging adulthood.

\section{Acknowledgments}

We thank the Family Studies Center at BYU, the School of Family Life, and the College of Family Home and Social Science at BYU, and we recognize the generous support of the many private donors who provided support for this project. We also thank those families who were willing to spend valuable hours with our team in interviews, and the many students who assisted in conducting the interviews.

\section{Appendix A. Supplementary data}

Supplementary data to this article can be found online at https://doi. org/10.1016/j.chb.2019.106160.

\section{References}

Andrews, S., Ellis, D. A., Shaw, H., \& Piwek, L. (2015). Beyond self-report: Tools to compare estimated and real-world smartphone use. PLoS One, 10(10), e0139004.

Banjanin, N., Banjanin, N., Dimitrijevic, I., \& Pantic, I. (2015). Relationship between internet use and depression: Focus on physiological mood oscillations, social networking and online addictive behavior. Computers in Human Behavior, 43, 308-312. https://doi.org/10.1016/j.chb.2014.11.013.

Barry, C. T., Sidoti, C. L., Briggs, S. M., Reiter, S. R., \& Lindsey, R. A. (2017). Adolescent social media use and mental health from adolescent and parent perspectives. Journal of Adolescence, 61, 1-11. https://doi.org/10.1016/j.adolescence.2017.08.005.

Berry, D., \& Willoughby, M. T. (2016). On the practical interpretability of cross-lagged panel models: Rethinking a developmental workhorse. Child Development, 88(4), 1186-1206. https://doi.org/10.1111/cdev.12660.

Best, P., Manktelow, R., \& Taylor, B. (2014). Online communication, social media and adolescent wellbeing: A systematic narrative review. Children and Youth Services Review, 41, 27-36. https://doi.org/10.1016/j.childyouth.2014.03.001.

Boers, E., Afzali, M. H., Newton, N., \& Conrod, P. (2019). Association of screen time and depression in adolescence. JAMA Pediatrics. https://doi.org/10.1001/ jamapediatrics.2019.1759 [Epub ahead of print].
Bulut, Z. A., \& Dogan, O. (2017). The ABCD typology: Profile and motivations of Turkish social network site users. Computers in Human Behavior, 67, 73-83. https://doi.org/ 10.1016/j.chb.2016.10.021.

Charles, S. (2019). Social media linked to rise in mental health disorders Accessed online at: https://www.nbcnews.com/health/mental-health/social-media-linked-rise-men tal-health-disorders-teens-survey-finds-n982526.

Coyne, S. M., Padilla-Walker, L. M., \& Howard, E. (2013). Emerging in a digital world: A decade review of media use, effects, and gratifications in emerging adulthood. Emerging Adulthood, 1, 125-137. https://doi.org/10.1177/2167696813479782.

Coyne, S. M., Padilla, W. L. M., Holmgren, H. G., \& Stockdale, L. A. (2019). Instagrowth: A longitudinal growth mixture model of social media time use across adolescence. Journal of Research on Adolescence. https://doi.org/10.1111/jora.12424.

Frison, E., \& Eggermont, S. (2017). Browsing, posting, and liking on Instagram: The reciprocal relationships between different types of Instagram use and adolescents' depressed mood. Cyberpsychology, Behavior, and Social Networking, 20(10), 603-609. https://doi.org/10.1089/cyber.2017.0156.

Hankin, B. L. (2006). Adolescent depression: Description, causes, and interventions. Epilepsy and Behavior, 8, 102-114.

Houghton, S., Lawrence, D., Hunter, S. C., Rosenberg, M., Zadow, C., Wood, L., et al. (2018). Reciprocal relationships between trajectories of depressive symptoms and screen media use during adolescence. Journal of Youth and Adolescence, 47(11), 2453-2467. https://doi.org/10.1007/s10964-018-0901-y.

Huang, C. (2018). Time spent on social networking sites and psychological well-being: A meta-analysis. Cyberpsychology, Behavior, and Social Networking, 20, 346-354. https://doi.org/10.1089/cyber.2016.0758.

Katz, E., Blumler, J. G., \& Gurevitch, M. (1974). Utilization of mass communication by the individual. In J. G. Blumler, \& E. Katz (Eds.), The uses of mass communications: Current perspectives on gratifications research (pp. 318-330). Beverly Hills, CA: Sage.

Lin, C. A. (1993). Exploring the role of VCR use in the emerging home entertainment culture. Journalism Quarterly, 70, 833-842. https://doi.org/10.1177\%2F107769909 307000409.

Lin, Y. H., Lin, Y. C., Lee, Y. H., Lin, P. H., Lin, S. H., Chang, L. R., \& TB, Kuo (2015). Time distortion associated with smartphone addiction: Identifying smartphone addiction via a mobile application (App). Journal of Psychiatric Research, 65, 139-145.

Lin, L. Y., Sidani, J. E., Shensa, A., Radovic, A., Miller, E., Colditz, J. B., et al. (2016). Association between social media use and depression among U.S. young adults. Depression and Anxiety, 33(4), 323-331. https://dx.doi.org/10.1002\%2Fda.22466.

Meyer, M., Adkins, V., Yuan, N., Weeks, H. M., Chang, Y., \& Radesky, J. (2019). Advertising in young children's apps: A content analysis. Journal of Developmental and Behavioral Pediatrics, 40, 32-39. https://doi.org/10.1097/ DBP.0000000000000622.

Nesi, J., Miller, A. B., \& Prinstein, M. J. (2017). Adolescents' depressive symptoms and subsequent technology-based interpersonal behaviors: A multi-wave study. Journal of Applied Developmental Psychology, 51, 12-19. https://doi.org/10.1016/j. appdev.2017.02.002.

Orben, A., \& Przybylski, A. (2019). The association between adolescent well-being and digital technology use. Nature Human Behavior, 3, 173-182. https://doi.org/ 10.1038/s41562-018-0506-1.

Petersen, A. C., Compas, B. E., Brooks-Gunn, J., \& Stemmler, M. (1993). Depression in adolescence. American Psychologist, 48, 155-168.

Quan-Hasse, A., \& Young, A. L. (2010). Uses and gratifications of social media: A comparison of Facebook and instant messaging. Bulletin of Science, Technology \& Society, 30, 350-361. https://doi.org/10.1177\%2F0270467610380009.

Rideout, V., \& Fox, S. (2018). Digital health practices, social media use, and mental wellbeing among teens and young adults in the U.S. San Francisco, CA: Hopelab and Well Being Trust. Available at: https://www.hopelab.org/reports/pdf/a-national-sur vey-by-hopelab-and-well-being-trust-2018.pdf.

Scherr, S., Toma, C. L., \& Schuster, B. (2018). Depression as a predictor of Facebook surveillance and envy: Longitudinal evidence from a cross-lagged panel study in Germany. Journal of Media Psychology: Theories, Methods, and Applications, 1-7. https://doi.org/10.1027/1864-1105/a000247.

Scott, H., \& Woods, H. C. (2018). Fear of missing out and sleep: Cognitive behavioural factors in adolescents' nighttime social media use. Journal of Adolescence, 68, 61-65. https://doi.org/10.1016/j.adolescence.2018.07.009.

Spence, S. H. (1998). A measure of anxiety symptoms among children. Behaviour Research and Therapy, 36, 545-566. https://doi.org/10.1016/S0005-7967(98) 00034-5.

Twenge, J. M. (2017a). IGen: Why today's super-connected kids are growing up less rebellious, more tolerant, less happy-and completely unprepared for adulthood-and what that means for the rest of us. New York: Simon and Schuster.

Twenge, J. M. (2017b). Have smartphones destroyed a generation? The Atlantic. September 2017 issue. Accessed online at: https://www.theatlantic.com/magaz ine/archive/2017/09/has-the-smartphone-destroyed-a-generation/534198/.

Twenge, J. M., Joiner, T. E., Rogers, M. L., \& Martin, G. N. (2018). Increases in depressive symptoms, suicide-related outcomes, and suicide rates among US adolescents after 2010 and links to increased new media screen time. Clinical Psychological Science, 6 (1), 3-17. https://doi.org/10.1177/2167702617723376.

Twenge, J. M., Martin, G. N., \& Campbell, W. K. (2018). Decreases in psychological wellbeing among American adolescents after 2012 and links to screen time during the rise of smartphone technology. Emotion, 18(6), 765-780. https://doi.org/10.1037/ emo0000403.

Vannucci, A., Flannery, K. M., \& Ohannessian, C. M. (2017). Social media use and anxiety in emerging adults. Journal of Affective Disorders, 207, 163-166. https://doi.org/ 10.1016/j.jad.2016.08.040.

Viner, R. M., Aswathikutty-Gireesh, A., Stiglic, N., Hudson, L. D., Goddings, A. L., Ward, J. L., et al. (2019). Roles of cyberbullying, sleep, and physical activity in mediating 
the effects of social media use on mental health and wellbeing among young people in england: A secondary analysis of longitudinal data. The Lancet Child \& Adolescent Health.

Weissman, M. M., Orvaschel, H., \& Padian, N. (1980). Children's symptom and social functioning self-report scales: Comparison of mothers' and children's reports. The
Journal of Nervous and Mental Disease, 168, 736-740. https://doi.org/10.1097/ 00005053-198012000-00005.

Woods, H. C., \& Scott, H. (2016). \#Sleepyteens: Social media use in adolescence is associated with poor sleep quality, anxiety, depression and low self-esteem. Journal of Adolescence, 51, 41-49. https://doi.org/10.1016/j.adolescence.2016.008. 